\title{
STATISTICAL SIGNAL PROCESSING FOR AUTOMOTIVE SAFETY SYSTEMS
}

\author{
Fredrik Gustafsson \\ Department of Electrical Engineering \\ Linköping University, SE-581 83 Linköping, Sweden \\ Email: fredrik@isy.liu.se
}

\begin{abstract}
The amount of software in general and safety systems in particular increases rapidly in the automotive industry. The trend is that functionality is decentralized, so new safety functions are distributed to common shared computer hardware, sensors and actuators using central data buses. This paper overviews recent and future safety systems, and highlights the big challenges for researchers in the signal processing area.
\end{abstract}

\section{INTRODUCTION}

Henry Ford revolutionized the automotive industry more than 100 years ago with his new production ideas. We are now facing another major shift in automotive production, when an increasing part of the value of the car comes from electronic systems. The introduction of more automotive safety systems plays an important role in this shift. For instance, one expert predicts that the value of software will increase from $4 \%$ in 2003 , to $13 \%$ in 2010 . This of course affects the engineering community in many ways. The automotive industry has always been dominated by mechanical engineers, but today we see an increasing need for engineers specialized in signal processing, automatic control, electronics, communication and computer hardware.

A key reason for this trend is the rapid development of safety systems. As the number of vehicles on our public roads increases, the requirement on safety is also increased. There has been a tremendous progress in this area over the last two decades as is evident from accident statistics. For instance, the number of fatalities in Sweden [12] suddenly started to drop around 1990. According to this report, the car fleet becomes safer for each year and the trend is that the fatality risk in a new car is reduced 5\% each year. A research report by an insurance company [11], partly acknowledges on-board safety systems for this trend change, and for instance it ranks an electronic stability system (antiskid control) as important as safety belts to prevent severe injuries on skiddy roads. The requirements on the safety systems will continue to increase in the future, motivating the continued development on improved versions of existing and new safety systems. The automotive executives share this view [23], as summarized in the conclusion "safety is a basic tenet to the industry now and will continue to be an ongoing major focus for consumers and manufacturers alike". Further, "new technology will be as important as new models in attracting customers".

The research community also has to contribute to this shift, and the purpose of this paper is to point out certain directions in signal processing where research is needed. The underlying theme is sensor fusion: to utilize existing and affordable sensors as efficiently as possible for as many purposes as possible. We point out certain more or less open problems in spectral analysis, non-uniform sampling, system identification, change detection, diagnosis and fault detection and model-based filtering and prediction to mention a few.

The outline of the paper is as follows. Section 2 overviews existing and some future control systems, where accurate information of the own and surrounding vehicles state is needed. Then, with this as a motivation, the following challenges are discussed:

Challenge I: Wheel speed analysis, using wheel speed as a generic multi-purpose sensor, is discussed in Section 3.

Challenge II: Virtual sensors for monitoring and control systems are discussed in Section 4.

Challenge III: Navigation as dynamic state estimation for monitoring and control systems is discussed in Section 5.

Challenge IV: Situational awareness by target tracking and road prediction for collision avoidance systems is discussed in Section 6.

Challenge V: Sensor-near signal processing, for improving and supplementing the sensor fusion, is discussed in Section 7.

Section 8 summarizes how sensor fusion may be structured in the future. 


\section{AUTOMOTIVE SAFETY SYSTEMS}

In this section, we will list and briefly present some different examples of new functions or systems being developed for or already in use in modern, high-end vehicles. The idea is to highlight that a rather small number of actuators and sensors would cover all functions, and any further new function could thus often be added as a pure software module.

Automotive safety systems can be grouped in many ways. The traditional way separates active safety (driving safety) systems that prevent accidents and passive safety (crash protection) systems that protect humans at an accident.

For passive safety, the signal processing opportunities are mainly in collision and roll-over detection for firing internal airbags and seat-belt pre-tensioners. Adaptive airbags for the passengers can fire at two or more levels. These systems require quite advanced signal processing and sensors mainly for classifying the passenger size and position. In the future, also external airbags and bonnet lifting for pedestrian protection systems $[25,15]$ may need advanced situational awareness.

However, the main challenges lie in active safety systems. These can be grouped into preventive systems (including driver information and driver drowsiness monitoring), dynamic control systems and collision avoidance systems. Some functions in the latter two categories, sometimes referred to as advanced driver assistance systems (ADAS, or just DAS) are summarized in Table 1. We here distinguish longitudinal control systems mainly used when driving straight ahead, lateral systems for cornering and maneuvering situations and rate control systems.

Table 1 illustrates that the main actuators are engine torque and brakes. The regulations currently say that the steered wheels must be physically connected to the steering wheel, thus steering is not currently used as an actuator. If these regulations change, several of the lateral control functions as yaw and roll control can be improved.

Here we would like to stress the following underlying statements for this survey:

1. Accurate state information is more important than advanced control algorithms (many of them are simple $\mathrm{P}(\mathrm{I}) \mathrm{D}$ controllers). That is, often it is more important what to feedback than how to feedback.

2. Using sensor fusion techniques, virtual sensors can be derived that compute information that would be either too costly or even impossible to measure in practice.

3. Model-based control systems are in their infancy, and future generations may include model-based filters and controllers.

The following five sections explain how this can be achieved by improved signal processing.

\section{CHALLENGE I: WHEEL SPEED ANALYSIS}

Without doubt, the wheel speed sensor is the potentially most informative sensor in the car. Today, the wheel speed is measured in all cars equipped with an anti-locking braking system (ABS) system on at least one but often all four wheels. Since the introduction of ABS control, wheel speed signal has found many other uses. The different potential applications presented in scientific literature and patents are c

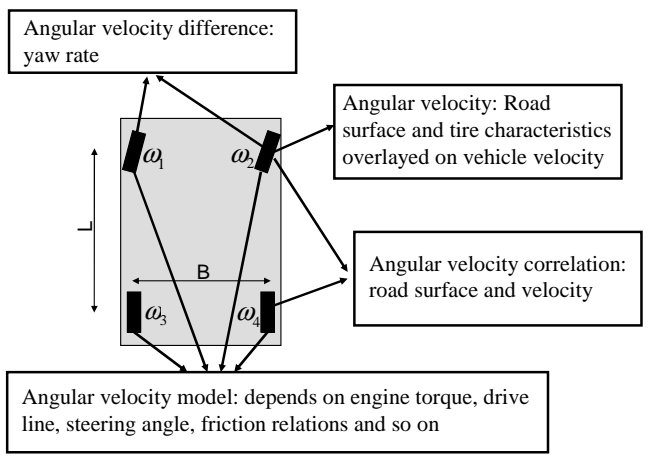

Fig. 1. Wheel speed applications.

First, the angular speeds relate to the vehicle speed at this tire by the tire radius. The speed content is a low frequency component. The tire dynamics can be modelled by three springs for torsional and radial forces in the tire rim, and the radial forces in the tire tread. Table 2 indicates the three modes. There are also wide-band noise induced from the road surface and low-frequency disturbance from the spring-damper system. Further, narrow-band disturbances as harmonics of the different rotating parts in the drive-line can sometimes be observed.

Examples of applications of the wheel speed information found in the literature (mainly patents) are illustrated in Figure 1 and include spectral analysis, correlation analysis and model-based approaches in signal processing.

\begin{tabular}{|c|c|c|c|c|c|c|}
\hline $0-10$ & $10-15$ & $15-30$ & $30-60$ & $60-80$ & $80-100$ & $100-$ \\
\hline Speed & Mode 1 & Noise & Mode 2 & Noise & Mode 3 & Noise \\
\hline Damper & \multicolumn{7}{|c|}{ Narrow-band noise components } & \\
\hline
\end{tabular}

Table 2. Frequency spectrum for the wheel speed signal, with approximate limits in $\mathrm{Hz}$.

\section{CHALLENGE II: VIRTUAL SENSORS}

A virtual sensor [19] is here defined as a physical quantity not directly measured, which is computed from existing sensors. The reason could be to avoid costly sensors, as in the tire pressure monitoring system in Section 4.2, or to 


\begin{tabular}{|c|c|c|c|c|c|c|c|c|c|}
\hline \multirow[b]{2}{*}{ System } & \multicolumn{4}{|c|}{ Actuator } & \multicolumn{5}{|c|}{ Primary sensors } \\
\hline & Brake & Acc. & Steering & Driver & Speed & IMU & DME & Brake & Vision \\
\hline Anti-locking Brake Systems (ABS) & $\overline{\mathrm{X}}$ & 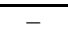 & 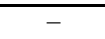 & 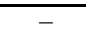 & $\overline{\mathrm{X}}$ & $\overline{-}-$ & $\overline{-}-$ & $\overline{\mathrm{X}}$ & 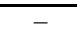 \\
\hline Brake Assistance Systems (BAS) & $\mathrm{x}$ & - & - & - & - & - & - & $\mathrm{x}$ & - \\
\hline Cruise Control & - & $\mathrm{x}$ & - & - & $\mathrm{x}$ & - & - & - & - \\
\hline Adaptive Cruise Control (ACC) & - & $\mathrm{x}$ & - & - & $\mathrm{x}$ & - & $\mathrm{x}$ & - & - \\
\hline Stop and Go & $\mathrm{x}$ & $\mathrm{x}$ & - & - & $\mathrm{x}$ & - & $\mathrm{x}$ & - & - \\
\hline Anti-spin control $(v<20 \mathrm{~km} / \mathrm{h})$ & $\mathrm{x}$ & - & - & - & $\mathrm{x}$ & - & - & - & - \\
\hline Traction control $(v>20 \mathrm{~km} / \mathrm{h})$ & - & $\mathrm{x}$ & - & - & $\mathrm{x}$ & - & - & - & - \\
\hline Forward Collision Warning (FCW) & - & - & - & $\mathrm{x}$ & $\mathrm{x}$ & - & $\mathrm{x}$ & - & - \\
\hline Forward Collision Mitigation (FCM) & - & - & - & - & $\mathrm{x}$ & - & $\mathrm{x}$ & - & - \\
\hline Parking aid systems & - & - & - & $\mathrm{x}$ & - & - & $\mathrm{x}$ & - & $\mathrm{x}$ \\
\hline Forward Collision Avoidance (FCA) & $\overline{\mathrm{X}}$ & - & $\overline{\mathrm{x}}$ & $\overline{-}$ & $\mathrm{x}$ & - & $\bar{x}$ & $\overline{-}-$ & $\overline{-}-$ \\
\hline Lane Keeping Aid systems (LKA) & - & - & $(\mathrm{x})$ & $\mathrm{x}$ & $\mathrm{x}$ & - & $\mathrm{x}$ & - & $\mathrm{x}$ \\
\hline Lane Change Aid systems & - & - & - & $\mathrm{x}$ & - & - & $\mathrm{x}$ & - & $\mathrm{x}$ \\
\hline Adaptive steering & - & - & $\mathrm{x}$ & - & $\mathrm{x}$ & - & - & - & - \\
\hline Yaw control & $\overline{\mathrm{X}}$ &  & $\overline{-}$ & - & $\overline{\mathrm{X}}$ & $\bar{x}$ & 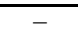 & $\overline{-}$ & - \\
\hline Roll stability control & $\mathrm{x}$ & - & - & - & $\mathrm{x}$ & $\mathrm{x}$ & - & - & - \\
\hline Roll-over detection (airbag) & - & - & - & - & $\mathrm{x}$ & $\mathrm{x}$ & - & - & - \\
\hline
\end{tabular}

Table 1. Advanced driver assistance systems (ADAS) grouped by longitudinal control, lateral control and rate control, respectively. Driver is here considered an actuator controlled by a haptic warning (dashboard lamp, sound, vibration). IMU means an inertial measurement unit, comprising at least one accelerometer or gyroscope. Distance measuring equipment (DME) includes radar, lidar and sonar measuring range and angle to objects. Vision includes camera and IR sensors.

compute abstract quantities as tire road friction as in Section 4.1 , where practically feasible sensors do not exist.

\subsection{Road friction monitoring}

A friction information system would be central for all dynamic control systems in Table 1, and would provide valuable information for the driver. The literature on this subject is quite rich, see the survey [28], and a multitude of approaches have been investigated:

- Slip based approaches, utilizing the noticable change in wheel slip (both lateral and longitudinal) with tire road friction [17].

- Spectral analysis of wheel speeds, as discussed in Section 3.

- Audio-based classification based on microphones 'listening' to each tire [35].

- Vision-based classification based on surface texture and reflection properties [9].

- Behavioural approaches analysing the driver behaviour [9], assuming the driver is aware of the friction and adapts his driving style. This is of course not an approach for monitoring, but can be used for initializing ABS control for instance.

- Sensor-based approaches where the sensors are mounted in the tires' tread for measuring stress [9].
Despite these efforts, no friction monitoring system exists on the market. Friction is a complicated physical phenomenon, and the variety of tire models and possible combination of makes and wear has delayed the introduction of large scale cheap commerical friction estimators substantially. Accurate and robust friction estimation may require multiple approaches with an over-all information fusion. Basically, all the approaches listed above can be used to obtain a more robust estimate.

\subsection{Tire pressure monitoring systems (TPMS)}

The following quote from the NHTSA press release from April 7, 2005, indicates the importance of development of reliable and affordable systems for tire pressure monitoring:

All passenger cars will have tire pressure monitoring systems beginning with the 2006 model year according to a new motor vehicle safety standard by the National Highway Traffic Safety Administration (NHTSA). The regulation will require that manufacturers install a system that can detect when one or more of the vehicle's tires are 25 percent or more below the recommended inflation pressure. Phase-in of the new regulation will begin Sept. 1, 2005. All new 4 -wheeled vehicles weighing 10,000 pounds or less must be equipped with the monitoring system by the 2008 model year.

Further,

NHTSA estimates that about 120 lives a year will be saved when all new vehicles are equipped 
with the tire pressure monitoring systems. In addition, consumers should see improved fuel economy and increased tire life.

See http://www.nhtsa.dot.gov/cars/rules/rulings for more details. The user interface is suggested to be as in Figure 2.

Today, most TPMS are sensor based [8, 1], using a pressure, temperature and possibly acceleration sensor in each wheel, each sensor kit equipped with a radio transmitter and battery. This is perhaps more of a sensor technology challenge.

However, there are indirect systems as well utilizing the wheel speeds at a much lower cost. More advance methods make use of sensor fusion on different scales. First, wheel speed analysis as described in Section 3 may indicate tire pressure information. Second, model-based sensor fusion can improve the estimation of relative tire radii substantially. High-level fusion of different principles may in the end enable a TPMS that satisfies the NHTSA rules.

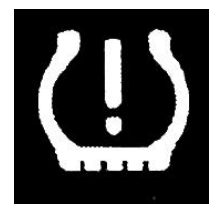

Fig. 2. The TPMS warning symbol.

\section{CHALLENGE III: DYNAMIC STATE ESTIMATION}

The dynamic control systems in Table 1 need accurate state information about the vehicle's position and orientation and their time derivatives. This is in general a model based filtering problem where the (extended) Kalman filter applies, where a partial vehicle model is used to improve sensor measurements and for diagnosis. We will here describe two non-standard problems.

Examples of application include [18]

- Offset-free velocity and acceleration (longitudinal dynamics) for cruise control and collision mitigation [37]

- Offset-free yaw rate and drift-free yaw angle (lateral dynamics) that can be used for ESP, lane keeping aid and navigation.

- Offset-free roll angle estimation (roll dynamics) for use with motorcycles (ABS, anti-spin, headlight control) or offset-free roll rate for vehicles (air bag curtains and roll-over detection).
- Positioning support and backup for satellite based positioning as the Global Positioning System (GPS) [13], see Figure 3. This includes improved dead-reckoning with small drift.

Sensor cost reduction and model based control system may necessitate further signal processing advances in this area.



Fig. 3. Positioning without GPS, using the particle filter based on wheel speed measurements and road map only, here implemented in a handheld computer.

\section{CHALLENGE IV: SITUATIONAL AWARENESS}

In particular collision avoidance systems and adaptive cruise controllers need advanced situational awareness, and short time prediction of the road and other vehicles' motion is crucial for safety related systems. Pre-crash systems, such as belt pre-tensioners and pedestrain protection systems, need accurate predictions and an estimate of the type of object of an imminient collision. Further, future engine control systems may need a road prediction for optimizing fuel economy and for automatic gear shifting.

The main sensors needed for situational awareness are one or a combination of radar [27], laser scanner [29, 15], lidar ([34] describes a 3D version), IR (including so called thermopiles [25]) and vision [31].

We split the situational awareness into the following subproblems:

- Classical target tracking problems, including Object recognition and the data association problem, which are more or less similar problems to what is studied in the area of air traffic control [4].

- Tracking and prediction of ego-motion (estimation of host vehicle state).

- Road geometry tracking and prediction. 
- Decision algorithms for warning and intervention systems.

Most publications in this field are concerned with target tracking and object recognition, describing sensor fusion with a subset of the sensors listed above: Sensor fusion of laser scanner and vision in the European Union project PROTECTOR is described in [21], while [16] also includes radar for pedestrian protection within the same project. Sensor fusion with focus on pedestrians is also treated in [26]. A Bayesian framework for sensor fusion is described in [6, 7], within the EU project CARSENSE. In the same project, [24] focuses on low speed situational awareness. Fusion of radar and IR is described in [32] and [30] fuses laser scanner and vision. Sensor fusion with focus on lateral tracking is described in [22] based on GPS, vision and radar and in $[3,2]$ based on vision and radar.

An ACC system based on inter-vehicle communication is described in [36]. Sensor requirements for a stop and go ACC system is discussed in [20, 14, 15].

A straightforward decentralized implementation of filters for situational awareness might look like the upper structure in Figure 4. The filters all need motion models for the road geometry, the ego-motion, the motion of tracked vehicles (often simpler than for ego-motion), and even a separate model for decision support.

However, road geometry and vehicle movements are highl: correlated, and further dependent on the type of vehicle (motorcycles have fast dynamics, bikes move slowly and close to the road side, etc.). More specifically, a lateral movement of tracked vehicles can be due to either a lane change or road bend, but a yaw change in the host vehicle gives quite similar sensor signals from the radar, lidar or vision sensor. Further, a displacement in two consequtive video frames of the radar bearing measurement from a stationary object may be used as a virtual yaw velocity sensor. See the left plot in Figure 5 for and illustration.

This motivates a centralized filtering approach according to the centralized filter in Figure 4. Here, the state vectors of the tracked vehicles and the road are merged into one state vector. In this way, movements of the tracked vehicles influence the road geometry estimate and vice versa.

The idea in centralized filtering is to merge all states in the models for road geometry, ego-motion and tracked vehicles into one state vector, cross-utilizing all measurements. One such model was developed independently in [10] and [22].

\section{CHALLENGE V: SENSOR-NEAR SIGNAL PROCESSING}

A radar system transmits a pulse and thresholds the response to get a range detection at each scanned bearing. The idea of "track before detect" in the target tracking literature [5,

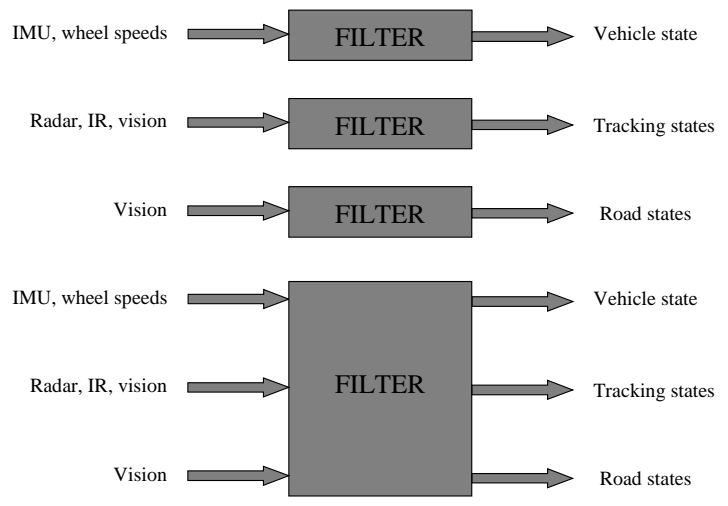

Fig. 4. Decentralized versus centralized filtering.

$33]$, is to measure the received signal energy in each rangebearing(-doppler) cell of the radar. In this way, a 2D (3D) image is obtained from the received waveform, as illustrated in the right plot in Figure 5. This can be used for estimating the spatial size of the object and its reflectance, which further is very useful for object classification. One of the few automotive publications on this approach [25] introduces the term "occupancy grid" thermopile sensors.
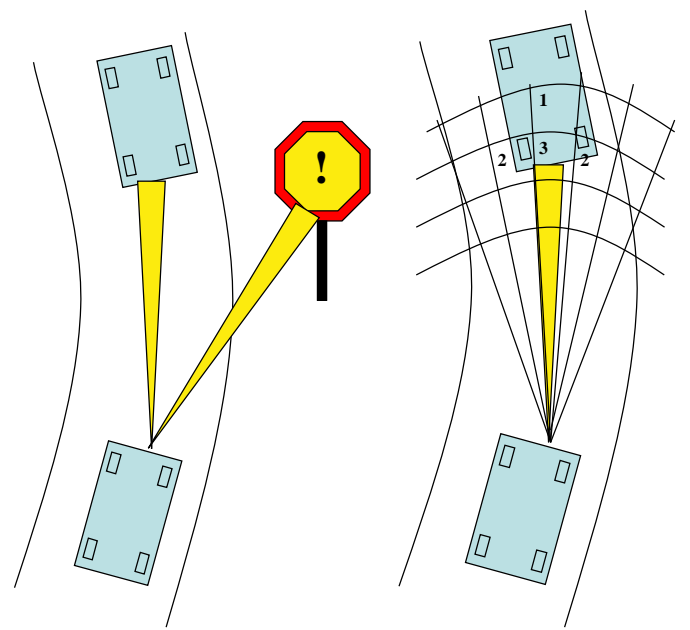

Fig. 5. Left: Radar as a sensor. Responses from moving objects are used for tracking and from stationary objects they can be used as inertial measurements. Right: The "track before detect" concept, where the received radar energy in each range-bearing cell is measured, rather than thresholded (detected).

Further, camera based vision systems may be used as both a tracking and an inertial sensor. One basic output from image processing is a list of features. In signal processing terms, each feature is an implicit non-linear measurement of the relative position to other moving objects (threats) and stationary objects. The former is the standard use of cameras in automotive safety systems, but features from station- 
ary objects along the road hide an unexplored potential use as a virtual sensor of velocity and yaw, pitch and roll rate.

\section{CONCLUSIONS}

Automotive safety systems rely on accurate estimation of external and internal state variables as well as unobserved environmental parameters as friction and tire pressure. We have in this contribution surveyed the main needs for signal processing development and argued for a sensor fusion approach where all tasks are considered jointly. First, Section 2 summarized a number of safety systems, and it was pointed out that a limited number of sensors can be sufficient to implement a variety of safety systems. Second, the active development of improved communication networks enables new sensor fusion strategies.

Figure 6 extends Figure 4 and summarizes our view on sensor fusion for future automotive safety systems. All available sensor information is communicated to the centralized or distributed sensor fusion algorithm. This includes vision sensors (camera, radar, lidar, IR), an inertial measurement unit (IMU) with up to three accelerometers and three gyroscopes, satellite navigation, and, most importantly wheel speeds. The output from the sensor fusion include virtual sensor signals, navigation information, tracking information and road geometry for collision avoidance systems.

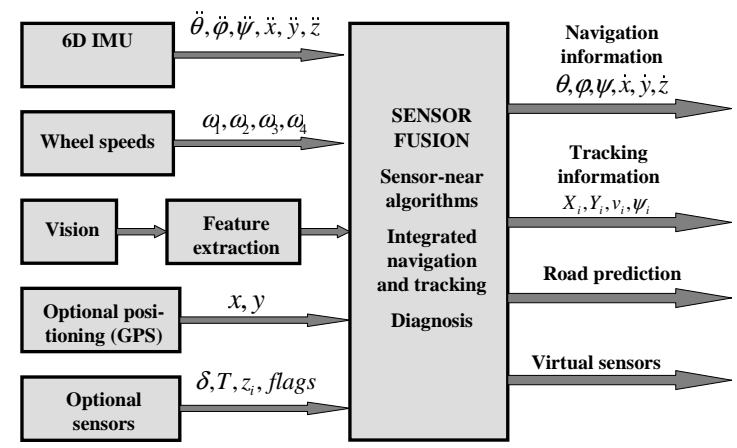

Fig. 6. Sensor fusion in future vehicles?

We have pointed out signal processing challenges ranging from low level sensor-near pre-processing to the highlevel algorithms for situational awareness. These are needed in advanced driver assistance systems for decision support, feedback control and driver alerts. Model-based control systems is one particular example, where signal processing design becomes particularly integrated with control system design.

\section{ACKNOWLEDGEMENT}

The long-term and extensive collaboration with NIRA Dynamics and Volvo Car Corporation is gratefully acknowledged. Dr. U. Forssell, CEO of NIRA and the many former and current students have substantially contributed to this work.

Grants from Vinnova's competence center ISIS and the IVSS (Intelligent Vehicle Safety Systems) program are also acknowledged.

\section{REFERENCES}

[1] National Highway Traffic Safety Administration. An evaluation of existing tire pressure monitoring systems. Technical Report DOT HS 809 297, US Department of Transportation, 2001.

[2] A. Amditis, N. Floudas, and A. Polychronopoulos. Lateral motion tracking of automobiles. In 7 th International Conference on Information Fusion, Stockholm, 2004.

[3] A. Amditis, A. Polychronopoulos, I. Karaseitanidis, G. Katsoulis, and E. Bekiaris. Multiple sensor collision avoidance system for automotive applications using an imm approach for obstacle tracking. In International Symposium on Information Fusion (IFIS), pages 812-817, 2002.

[4] Y. Bar-Shalom and X.R. Li. Estimation and tracking: principles, techniques, and software. Artech House, 1993.

[5] Y. Boers and J.N. Driessen. Particle filter based detection for tracking. In IEEE American Control Conference, volume 6, pages 4393-4397, Arlington, 2001.

[6] C. Coue, T. Fraichard, P. Bessiere, and E. Mazer. Multi-sensor data fusion using bayesian programming: an automotive application. In IEEE Int. Conf. on Intelligent Robots and Systems, Switzerland, 2002.

[7] C. Coue, T. Fraichard, P. Bessiere, and E. Mazer. Using bayesian programming for multi-sensor multitarget tracking in automotive applications. In IEEE Int. Conf. on Robotics and Automation (ICRA), Taipai, Taiwan, 2003.

[8] Apollo Deliverable D7. Intelligent tyre systems - state of the art and potential technologies. Technical report, EU-IST-2001-34372, 2003.

[9] U. Eichhorn and J. Roth. Prediction and monitoring of tyre/road friction. In Proceedings of FISITA, London, June 1992. 
[10] A. Eidehall and F. Gustafsson. Combined road prediction and target tracking in collision avoidance. In IEEE Intelligent Vehicle Symposium (IV'04), pages 619-624, Parma, Italy, 2004.

[11] Folksam. Hur säker är bilen? (How safe is the car?). Technical report, http://www.folksam.se/ resurser/pdf/sakrabilar2005.pdf, 2005.

[12] Swedish Institute for Transport and Communications Analysis Statistics Sweden. Road traffic injuries 2003, official statistics of sweden. Technical report, http://www.sika-institute. se/databas/data/ss_2004_6.pdf, 2004.

[13] U. Forssell, P. Hall, S. Ahlqvist, and F. Gustafsson. Novel map-aided positioning system. In Proc. of FISITA, number F02-1131, Helsinki, 2002.

[14] R. Fredriksson, Y. Håland, and J. Yang. Evaluation of a new pedestrain head injury protection system with a sensor in the bumper and lifting of the bonnet's rear part. In Enhanced Safety of Vehicles Conference, pages $1-11,2001$.

[15] K.C. Fürstenberg, D. Linzmeier, and U. Lages. Pedestrian recognition and tracking of vehicles using a vehicle based multilayer laser-scanner. In World Congress on Intelligent Transport Systems and Services (ITS), Madrid, 2003.

[16] D.M. Gavrila, M. Kunert, and U. Lages. A multisensor approach for the protection of vulnerable traffic participants - the protector project. In IEEE Instrumentation and Measurement Technology Conference, Budapest, 2001.

[17] F. Gustafsson. Slip-based estimation of tire - road friction. Automatica, 33(6):1087-1099, 1997.

[18] F. Gustafsson, S. Ahlqvist, U. Forssell, and N. Persson. Sensor fusion for accurate computation of yaw rate and absolute velocity. In Society of Automotive Engineers World Congress, number SAE 200101-1064, Detroit, 2001.

[19] F. Gustafsson, M. Drevö, U. Forssell, M. Löfgren, N. Persson, and H. Quicklund. Virtual sensors of tire pressure and road friction. In Society of Automotive Engineers World Congress, number SAE 200101-0796, Detroit, 2001.

[20] R. Hallouzi, V. Verdult, H. Hellendoorn, and J. Ploeg. Experimental evaluation of a co-operative driving setup based on inter-vehicle communication. In IFAC/EURON Symposium on Intelligent Autonomous Vehicles (IAV), 2004.
[21] N. Kaempchen, K.C. Fuerstenberg, A.G. Skibicki, and K.C.J. Dietmayer. Sensor fusion for multiple automotive active safety and comfort applications. In International Forum on Advanced Microsystems for Automotive Applications (AMAA), Berlin, 2004.

[22] A. Klotz, D. Hoetzer, and J. Sparbert. Lane data fusion for driver assistance systems. In 7th International Conference on Information Fusion, Stockholm, 2004.

[23] KPMG. Global auto executive survey. Automotive momentum, 1(1), 2005.

[24] J. Langheim and A.J. Buchanan. Carsense - sensing of car environment at low speed driving. In World Congress on Intelligent Transport Systems and Services (ITS), Turin, 2000.

[25] D. Linzmeier, M. Mekhaiel, J. Dickmann, and K.C.H. Dietmayer. Pedestrian detection with thermopiles using an occupancy grid. In World Congress on Intelligent Transport Systems and Services (ITS), Washington, 2004.

[26] P. Marchal, D. Gavrila, L. Letellier, M-M. Meinecke, R. Morris, and M. Töns. Save-u : Sensor data fusion for improved vulnerable road user detection. Budapest, 2004.

[27] C. Metz, J. Grubert, J. Heyen, A.F. Jacob, S. Janot, and E. Fully integrated automotive radar sensor with versatile resolution. IEEE Transactions on Microwave Theory and Techniques, 49(12):2560-2566, 2001.

[28] S. Müller, M. Uchanski, and K. Hedrick. Estimation of the maximum tire-road friction coefficient. Journal of Dynamic Systems, Measurement, and Control, 125:607-617, 2003.

[29] B. Nitsche and R. Schulz. Automotive applications for the alasca laserscanner. In International Forum on Advanced Microsystems for Automotive Applications (AMAA), Berlin, 2004.

[30] A. Oyaide, V. Willhoeft, C. Shooter, and A. Heenan. Lane and road detection using video and laserscanner data. In World Congress on Intelligent Transport Systems and Services (ITS), Madrid, 2003.

[31] H. Rehbinder and B.K. Ghosh. Pose estimation using line-based dynamic vision and inertial sensors. IEEE Transactions on Automatic Control, 48(2):186-199, 2003.

[32] M.E. Russell, C.A. Drubin, A.S. Marinilli, W.G. Woodington, and M.J. DelCheccolo. Integrated automotive sensors. IEEE Transactions on Microwave Theory and Techniques, 50(3), 2002. 
[33] D.J. Salmond and H. Birch. A particle filter for track before detect. In IEEE American Control Conference, volume 6, pages 4393-4397, Arlington, 2001.

[34] R.M. Schwarte. Breakthrough in multichannel laser-radar technology providing thousands of highsensitive lidar receivers on a chip. In International Society for Optical Engineering (SPIE), volume 5575, pages 126-136, 2004.

[35] A. Svärdström. Classification of road surface using a neural net (in swedish). Technical Report UPTEC 93 056R, Uppsala University, Uppsala, Sweden, June 1993.

[36] P. Venhovens, K. Naab, and B. Adiprasito. Stop and go cruise control. International Journal of Automotive Technology, 1(2):61-69, 2000.

[37] K. Watanabe, K.C. Cheok, and K. Kobayashi. Absolute speed measurement of automobile from noisy acceleration and erroneous wheel speed information. SAE Paper, 75-82, 1992. 